\title{
Migration of a marine oligochaete: induction of dispersal and microhabitat choice
}

\author{
Per G. Nilsson*, Jeffrey S. Levinton, Josepha P. Kurdziel \\ Department of Ecology and Evolution, State University of New York at Stony Brook, Stony Brook, New York 11794, USA
}

\begin{abstract}
We present evidence for migration in the asexual phase of the life cycle of Paranais litoralis (Müller), a marine oligochaete that reproduces asexually by fission. Depletion of resources triggers a swimming response by some worms ('migrators'). Migrating worms are longer, thinner and have more segments than non-migrating worms, indicating that migrating worms postpone reproduction. Experiments show that migrators swim more actively than non-migrators, and that this causes them to stay suspended in the water column longer than non-migrators. Worms avoid sediment where resources are exhausted, both when burrowing and when returning to the bottom after swimming. Based on laboratory cultures and field observations, we hypothesize that local depletion of resources is a common phenomenon for $P$. litoralis, and that migration is important for the persistence of $P$. litoralis populations.
\end{abstract}

KEY WORDS: Migration - Swimming $\cdot$ Colonization $\cdot$ Asexual reproduction $\cdot$ Spatial population model

Resale or republication not permitted without written consent of the publisher -

\section{INTRODUCTION}

The spatial dynamics of an organism may influence the biology of an individual and of the population in many ways (Dias 1996, Harrison \& Hastings 1996, Palmer et al. 1996, Sugg et al. 1996). Several authors have noted that species living in ephemeral habitats tend to migrate more than species living in stable habitats (e.g., Dingle 1996, Palmer et al. 1996). When migrators reach a new patch, they may remain there to start a new population. Although populations may have a positive growth rate once established, demographic stochasticity during the establishment phase following immigration may lead to frequent extinction (Nunney \& Campbell 1993). This may explain the absence of species from an area, despite suitable habitat and adequate dispersal capability. Two ways of decreasing the risk of stochastic demographic extinction are to grow fast (short generation time and high fecundity) and to escape the need for a sexual partner. These are charac-

*Present address: Tjärnö Marine Biological Laboratory, 45296 Strömstad, Sweden. E-mail: per.nilsson@tmbl.gu.se teristics of most asexual organisms, and (facultatively) asexual organisms are common in ephemeral habitats (Hughes 1989). However, asexual reproduction may also have the potential disadvantage of not producing a resting and/or migratory stage (Hughes 1989). Many clonal organisms reproduce asexually while they can expand locally, but when all locally available space or resource is used they produce sexual offspring that constitute the migratory stage. It is, however, not necessary for the migratory stage to be sexual: a parthenogenetically produced offspring in the form of a migratory larva would still give the advantage of asexual reproduction. The fact that the migratory stage is so frequently sexual suggests the importance of genetic variation in the offspring, presumably because the larvae may encounter conditions different from those experienced by the mother. If, however, the adverse conditions triggering the response to migrate are likely to be spatially restricted, then the conditions at the place where the migrant settles may still be similar to the conditions experienced by the mother, and an asexual dispersal phase may be more advantageous than a sexually produced offspring. 
Oligochaetes frequently occur in ephemeral and disturbed habitats, which suggests the presence of an efficient dispersal mechanism. However, many species of oligochaetes reproduce asexually by fission, especially in the families Naididae and Aeolosomatidae (Lasserre 1975). When they reproduce sexually, it is by insemination (possibly reciprocal) and by the formation of cocoons (Lasserre 1975, Giere \& Pfannkuche 1982). The offspring emerge from the cocoon as a smaller but morphologically similar version of the parents, i.e., oligochaetes lack a planktonic larval migratory stage. For example, the naidid oligochaete Paranais litoralis (Müller) is common worldwide in estuarine habitats, especially intertidal mud flats (Giere \& Pfannkuche 1982). They typically undergo a pronounced yearly cycle, with low abundance during winter, increasing population densities during spring, reaching maximum population densities in early summer, after which the populations crash and occur only at low densities until the following spring (Giere \& Pfannkuche 1982, Cheng et al. 1993, Cheng \& Chang 1999). Sometimes a second but smaller population increase is found in autumn. The population distribution is patchy during peak numbers (Nilsson et al. 1997). Laboratory cultures have shown that there are differences in nutritional quality of the sediment both among seasons and among places within seasons (Cheng et al. 1993, Nilsson et al. 1997), consistent with the patchy field occurrence of the oligochaetes. The typical asexual offspring produced by $P$. litoralis is an almost identical copy of the mother: in laboratory cultures, the offspring matures within about $4 \mathrm{~d}$ to produce its first offspring (Martinez 1993, Nilsson pers. obs.). Since no recombination is involved in this process, it may also be seen as growth, although we use the term asexual reproduction here to emphasize that a new detached 'unit' is produced (Hughes 1989). We have never observed sexual reproduction in $P$. litoralis in our laboratory cultures, or sexually mature worms in field samples. $P$. litoralis is also a typical oligochaete in that it lacks a larval dispersal stage. However, we have frequently observed adult individuals of $P$. litoralis swimming in our high-density laboratory cultures. This suggested to us that swimming might be a response by the worms to adverse conditions, which may have important effects on the population biology of $P$. litoralis if it occurs in nature. Many investigators have reported normally infaunal meiofauna-sized animals in the water column (Giere 1993). Several passive or active triggering mechanisms for water-column dispersal have been suggested and tested, including hydrodynamic conditions, tidal cycle, and high population density (Palmer 1988).

In this investigation, we test if swimming by Paranais litoralis is a specialized migration behavior and what the consequences of migration are for the population biology of the species. Our goal is to link individual behavior to population processes, a field of study we believe is particularly important in spatially divided populations. The study has been done on a species in a group (oligochaetes) that, to our knowledge, has not been previously studied from this point of view, but that shares a common habitat (intertidal mud flats) and mode of living (endobenthic burrowers) with many types of organisms around the world. Through a series of laboratory experiments and field observations of $P$. litoralis, we examine the interaction between migration and the morphology and behavior of individuals. We address the following main questions: (1) What proximate factors cause migration by swimming, and can migrators choose where to settle? (2) Do migrators differ morphologically, physiologically and behaviorally from non-migrators?

\section{METHODS}

Stock cultures. Stock cultures of Paranais litoralis were started in June 1993 from individuals collected in Flax Pond, an intertidal mud flat surrounded by a Spartina alterniflora marsh on the north shore of Long Island, New York, USA. New worms were collected from the field on several occasions during 1993 and 1994, and added to the stock cultures. Cultures were kept at $13^{\circ} \mathrm{C}$ in a flow-through seawater system, in sediment collected from Flax Pond. Sediment was frozen and kept at $-20^{\circ} \mathrm{C}$ until use. New sediment was collected every month to ensure good sediment quality.

Induction of migration. Long-term induction experiment: This experiment was designed to investigate whether migration by swimming was induced by density or by the depletion of resources. We added $3 \mathrm{ml}$ of frozen and thawed Flax Pond spring sediment and 5 worms from the stock cultures to each of 6 glass bowls (diameter $55 \mathrm{~mm}$ ). The bowls had a rim that extended about $1 \mathrm{~cm}$ above the sediment surface, so that worms had to swim in order to escape from the bowls. The bowls were put individually in aquaria with filtered seawater, and kept at $18^{\circ} \mathrm{C}$. Under these culture conditions, worm numbers may double every $4 \mathrm{~d}$ if food is superabundant (Nilsson et al. 1997). There were 2 treatments: resource renewal and resource depletion. Three of the bowls received new sediment at each check and therefore should not have experienced resource depletion (but see 'Discussion'); the other 3 bowls received the original sediment so that resources would be depleted with time. Every $3 \mathrm{~d}$ we counted the number of worms in the bowls and the number of worms on the bottom of the aquaria. The worms outside the bowls were called migrants. On 3 
occasions (Days 10, 17 and 27), 10 to 25 randomly chosen worms from each treatment (sediment renewal and depletion) and of each category (migrants and non-migrants), i.e., a total of about 40 to 100 worms per occasion, were measured (see below). The experiment was allowed to run until all worms in the resource depletion treatment were dead (30 d). To check that migration was not induced by the buildup of excretion products in the sediment, we washed the sediment in the resource depletion treatment in ca $50 \mathrm{ml}$ filtered seawater each time the worms were checked.

Short-term induction experiment: We also tested if nutrient-poor sediment would induce migration in worms in good nutritional status. We took the sediment from the resource depletion treatment, rinsed it with filtered seawater several times, put it in 5 bowls (diameter $55 \mathrm{~mm}$ ), and allowed it to stand in seawater with aeration for $1 \mathrm{wk}$. New Flax Pond sediment (frozen and thawed) was added to 5 other bowls which were treated identically. After $1 \mathrm{wk}$, the bowls were transferred to individual aquaria and 10 worms from the stock cultures were added to each bowl. The number of migrant and non-migrant worms was then counted after 2 and $5 \mathrm{~d}$ without changing the sediment.

Survival and reproduction of migrators. This experiment was designed to investigate possible differences in life history characteristics between migrant (swimming) and non-migrant worms. In the long-term induction experiment described above, 2 additional bowls where sediment was not exchanged were set up in individual trays. After $18 \mathrm{~d}$, all worms found in the trays (18 swimming worms found outside the bowls and 14 burrowing worms found in the bowls) were transferred individually to bowls with new Flax Pond sediment (frozen and thawed) and cultured under conditions as described above. These cultures were checked every $2 \mathrm{~d}$ for $12 \mathrm{~d}$, and survival and the number of worms producing offspring were recorded.

Selection of sediment by swimming worms. This experiment was designed to investigate if migrating (swimming) worms would choose where to settle based on sediment quality. In each of 5 replicate aquaria, 5 bowls were set up: 2 containing $3 \mathrm{ml}$ of frozen and thawed sediment from Flax Pond ('good' sediment), 2 containing sediment kept from previous experiments where worm populations had exhausted the resources ('exhausted' sediment), and 1 bowl with a 1:1 mix of the 2 kinds of sediment. The positions of the bowls were randomly varied among aquaria. Worms (15 individuals from the stock cultures) were added to the 1:1mix bowl (placed in the center of the aquarium). The number of worms in each bowl and in the surrounding aquarium was checked every $2 \mathrm{~d}$ for $1 \mathrm{wk}$.

Selection of sediment by burrowing worms. This experiment was designed to investigate if worms choose patches of sediment based on sediment quality on a smaller spatial scale by means of burrowing. In each of 4 replicate aquaria, frozen and thawed sediment was added to a large petri dish (diameter $14 \mathrm{~cm}$ ). Each dish contained 6 sediment patches (patch diameter $=25 \mathrm{~mm}$ ): 3 where the sediment was replaced with nutrient-exhausted sediment and 3 where sediment was removed and then returned, to control for the disturbance of the sediment ('good' sediment). The arrangement of patches was randomly varied among aquaria. The dishes were left undisturbed for $24 \mathrm{~h}$. At the start of the experiment, 100 worms from stock cultures were added to the center of each dish. After $48 \mathrm{~h}$, the number of worms in each patch was counted.

Morphology of migrators and non-migrators. Migrating and non-migrating worms were transferred to petri dishes filled with filtered seawater and filmed at $20 \times$ with a video camera attached to a dissecting microscope. The video images were transferred to a Macintosh PowerPC 8100/AV and measured for length and projected area using the public domain program NIH Image Version 1.55 (Wayne Rasband, US National Institute of Health). Average width was calculated as the projected area divided by the length.

We counted the number of segments to investigate if migrating and non-migrating worms differed in the number of segments. Fifteen migrating and nonmigrating worms from the long-term induction experiment were transferred individually to vials and fixed in $70 \%$ alcohol. The number of segments bearing setae was checked under a compound microscope at $200 \times$.

Swimming activity and sinking rates of worms. We placed worms from the migration-induction experiment (migrators and non-migrators) $100 \mathrm{~mm}$ above the bottom of a $0.5 \mathrm{l}$ glass beaker (diameter $87 \mathrm{~mm}$ ) filled with Flax Pond water. We recorded the time until the worm reached the bottom of the beaker and the proportion of this time spent swimming. We also measured the length and width of these worms as described above.

Diffusion experiments. We added sediment (frozen and thawed) to petri dishes (diameter $14 \mathrm{~cm}$ ) in a $3 \mathrm{~mm}$ layer. The petri dishes were put in aquaria with Flax Pond water and left for $24 \mathrm{~h}$. The next day, 50 nonswimming worms from the stock cultures were added to the center and allowed to move for 15 to $30 \mathrm{~min}$. We then stopped the experiment, and the sediment was collected with a pipette from 5 concentric rings. The distance traveled by a worm found in one of these rings was calculated as the geometric average of the distance from the center of the dish to the inner and outer boundary of that ring. The 2-dimensional diffusion constant $D\left(\mathrm{~mm}^{2} \mathrm{~min}^{-1}\right)$ for the movement of worms was calculated as $D=M S D / 4 t$, where $M S D=$ mean square displacement $=$ the squared distance a worm moved from the point of origin, averaged over all 
worms, and $t=$ duration of the experiment (Okubo 1980, Berg 1993). The experiment was repeated on 5 occasions, each time with 2 new petri dishes and new worms. Diffusion coefficients for swimming worms were measured by adding 10 migrator worms to 3 replicate petri dishes as described above, and recording their movements as they were swimming.

Field occurrence of migration. Ten sediment samples were taken with a cut-off syringe (inner diameter $25 \mathrm{~mm}$ ) to a depth of $10 \mathrm{~mm}$ at random locations in Flax Pond during an outgoing tide (water depth 45 to $60 \mathrm{~cm}$ ), on May 31, 1995. Samples were preserved in $4 \%$ formalin solution with Rose Bengal added. We also took water samples at 12 randomly chosen locations. Each sample consisted of 121 of water, sieved through a $250 \mu \mathrm{m}$ nylon mesh. The residue collected on the mesh was preserved in $4 \%$ formalin. We counted the number of worms found in the sediment and water samples, and the number of segments on each worm was counted at $200 \times$ under a compound microscope.

Statistical analyses. Data were analyzed with 1-way and factorial ANOVA, and ANCOVA in SYSTAT for Macintosh, Version 5.2 (SYSTAT Inc., Evanston, Illi-
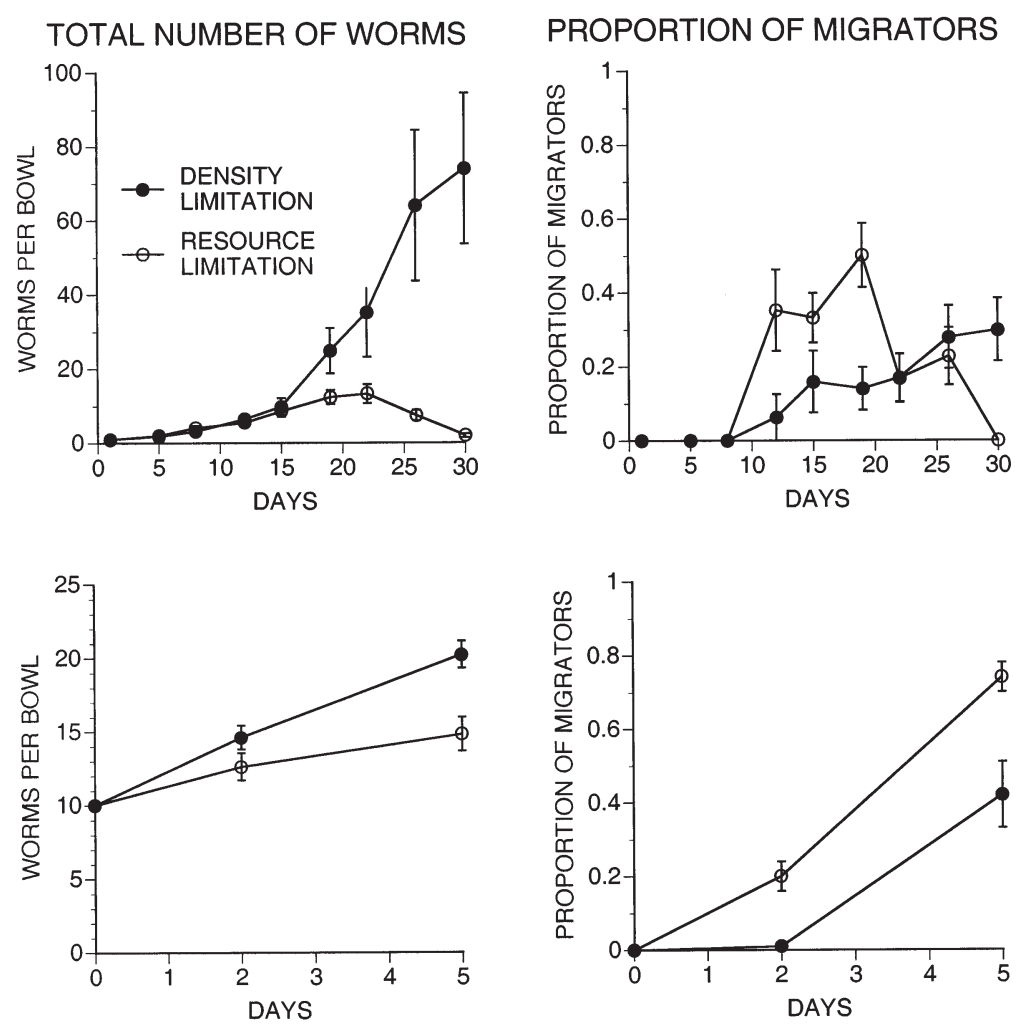

Fig. 1. Paranais litoralis. Number of worms and proportion of migrators in cultures where sediment was renewed (density limitation) and cultures where sediment was not renewed (resource limitation), in longterm experiment ( $30 \mathrm{~d}$, top panels) and in short-term experiment ( $5 \mathrm{~d}$, bottom panels). Mean $\pm \mathrm{SE}, \mathrm{n}=3$ nois, USA). Homogeneity of variances was tested with Cochran's test (Winer et al. 1991). Homogeneity of slopes in ANCOVA was tested by examining the interactions between the covariate and other factors. When repeated measures on the same subject were analyzed, the MANOVA option for repeated measures was used (von Ende 1993). Differences between migrators and non-migrators in survival and fecundity were tested with a $\log$-linear test for independence (G-test, Sokal \& Rohlf 1981).

\section{RESULTS}

\section{Induction of migration}

Our results indicate that exhaustion of resources, rather than density of Paranais litoralis alone, caused the emergence of migrating worms. The proportion of migrating worms was significantly higher in the resource depletion treatment compared to the resource renewal treatment (Fig. 1, repeated-measures ANOVA time $\times$ sediment interaction, $F_{4,19}=3.145, \mathrm{p}=0.038$ ), although the density of worms was consistently higher in the latter. After $10 \mathrm{~d}$, migrators also started to emerge in bowls where sediment was renewed. It is possible that $3 \mathrm{~d}$ between changes of sediment was too infrequent to prevent some resource depletion at the very high densities encountered near the end of the experiment, or density in itself may have caused migration at very high densities. The short-term induction experiment showed the same pattern (Fig. 1): worms in the resource depletion treatment migrated significantly more than worms in the sediment renewal treatment (repeated-measures ANOVA time $\times$ sediment interaction, $F_{2,7}=11.676$, $\mathrm{p}=0.006)$.

We found no significant differences between swimmers and non-swimmers in number of offspring per worm (migrators: $1.25 \pm$ 0.25 mean $\pm \mathrm{SE}, \mathrm{n}=18$; non-migrators: $1.0 \pm$ $0.33, \mathrm{n}=14 ; 1$-way ANOVA, $F_{1,22}=0.37$, $p=0.54$ ), proportion of worms reproducing, or in survival for the $12 \mathrm{~d}$ period that we followed individual worms (Fig. 2).

\section{Selection of sediment}

This experiment showed that the worms settled selectively in 'good' sediment after migration by swimming (Fig. 3). Already after $2 \mathrm{~d}$, the number of worms in 'good' (i.e., 

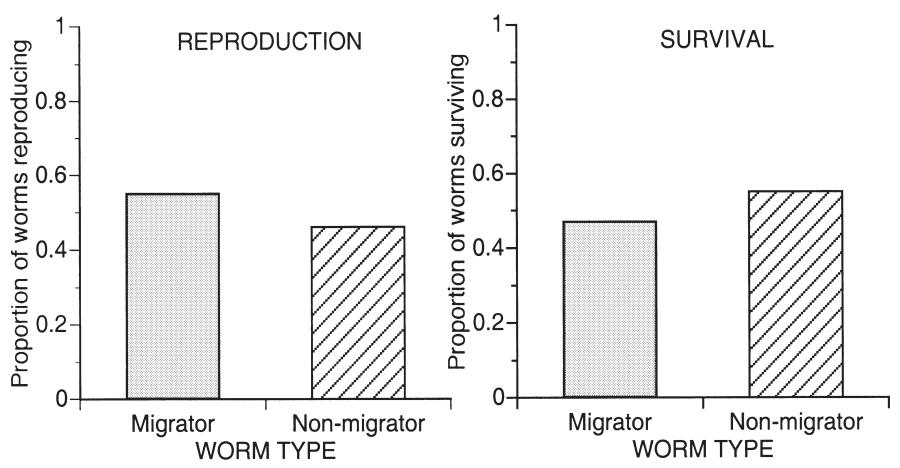

Fig. 2. Paranais litoralis. Survival and proportion of worms reproducing in migrators $(\mathrm{n}=18)$ and non-migrators $(\mathrm{n}=14)$ during a $12 \mathrm{~d}$ period following transfer from exhausted sediment to high-quality sediment. Differences among worm types are not significant ( $G$-test, $G=0.51, \mathrm{p}=0.47$ )

renewed) sediment was significantly higher than the number of worms in 'exhausted' sediment (2-way ANOVA sediment effect, $F_{1,4}=96.4, p=0.006$ ). Although some of this difference may have been caused by reproduction of worms in 'good' sediment, the time frame of the experiment was too short and the difference between treatments too great to be explained by reproduction alone. Furthermore, the number of worms found in the surrounding aquarium was much lower than would be expected if they had just settled in proportion to area. This indicates that worms swam actively until they found a suitable spot. Worms also chose favorable sediment when burrowing. Significantly more worms were found in spots with 'good' sediment than in spots with 'exhausted' sediment (Fig. 3, 2-way ANOVA sediment effect, $F_{1,3}=17.5$, $\mathrm{p}=0.025)$.
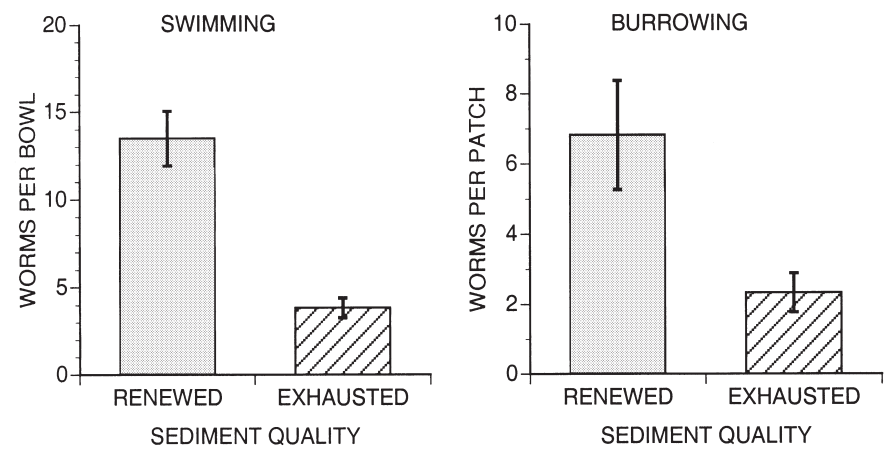

Fig. 3. Paranais litoralis. Sediment choice by worms while swimming and while burrowing. Bars show the average number of worms in bowls with renewed or exhausted sediment (left panel), or in patches of renewed or exhausted sediment (right panel) after $48 \mathrm{~h}$. Results are pooled for all trays. Means $\pm \mathrm{SE}, \mathrm{n}=10$ (swimming) and $\mathrm{n}=12$ (burrowing)
Table 1. Paranais litoralis. Width of worms. ANCOVA showing effect of worm type (migrator or non-migrator, fixed factor), culture condition (sediment renewal or not, fixed factor), and worm length (covariate)

\begin{tabular}{|lrccl|}
\hline Source of variation & df & MS & $F$ & $\mathrm{p}$ \\
\hline Worm type & 1 & 0.0051 & 6.18 & 0.014 \\
Culture condition & 1 & 0.0045 & 5.46 & 0.020 \\
Worm $\times$ Culture & 1 & 0.0006 & 0.70 & 0.40 \\
Length & 1 & 0.0047 & 5.66 & 0.018 \\
Residual & 232 & 0.0008 & & \\
\hline
\end{tabular}

\section{Morphological differences}

Worms found outside bowls (migrators) were on average significantly longer than worms that remained in bowls (1-way ANOVA, $F_{1,233}=13.7, \mathrm{p}<0.001$ ), and were thinner for a given length (Table 1), irrespective of the type of sediment. Migrating worms also had more segments $(36.87 \pm 2.20$, mean $\pm \mathrm{SE}, \mathrm{n}=15)$ than non-migrating worms $(26.0 \pm 1.49)(1$-way ANOVA, $\left.F_{1,28}=16.7, p=0.0003\right)$. Since the fission zone in Paranais litoralis normally occurs at Segment 18 (Martinez 1993), the average number of segments in migrants (37) suggests that migrating worms postpone fission when resources are in short supply and until they find suitable sediment after migrating.

\section{Swimming frequency and sinking rate}

Migrator worms stayed in the water column significantly longer than non-migratory worms (1-way ANOVA, $F_{1,115}=4.49, \mathrm{p}=0.036$ ). Two factors contributed to this: (1) migrators were on average longer than non-migrators, and longer worms sank slower than short worms; (2) migrators were more active swimmers than non-migrators. When the variation caused by these 2 factors is partitioned out, there is no significant difference between worm types (Table 2).

\section{Movements in sediment: diffusion experiments}

The diffusion coefficient for worms burrowing in sediment was $4.22 \pm 0.36 \mathrm{~mm}^{2} \mathrm{~min}^{-1}$ (mean $\pm \mathrm{SE}, \mathrm{n}=$ $10)$, while migrators swimming had a diffusion coefficient of $34.0 \pm 4.57(\mathrm{n}=3) \mathrm{mm}^{2} \mathrm{~min}^{-1}$.

\section{Occurrence of migration in the field}

Swimming worms were found in the water samples taken in spring of $1995(1.67 \pm 0.93$ per $12 \mathrm{l}, \mathrm{n}=12)$. At 
this time, worm densities in the sediment were $6.11 \pm$ 6.02 per $10 \mathrm{~cm}^{2}(\mathrm{n}=10)$. The number of segments per worm was significantly higher for worms found in water samples $(35.2 \pm 0.79$ segments, mean $\pm \mathrm{SE}, \mathrm{n}=$ 12) than for worms found in the sediment (24.6 \pm 0.95 segments, $\mathrm{n}=26$ ) (1-way ANOVA, $F_{1,36}=49.4, \mathrm{p}=$ $0.0001)$. Out of 26 worms, 2 found in sediment samples had $>30$ segments (both had 34 segments), while all worms found in the water samples had $>33$ segments. The number of segments of swimmers and non-swimmers in the field were not significantly different from swimmers and non-swimmers found in the laboratory.

\section{DISCUSSION}

This study on Paranais litoralis is an attempt to link the everyday behavior of individuals (feeding) to a specialized behavior (migration due to shortage of resources) and the population biology of the species. We focus our discussion on 3 points: (1) Are the swimming worms really migrators, i.e., is this a specialized behavior for moving to new habitats? (2) Is migration a favorable trait? (3) What are the potential consequences for the population biology of Paranais litoralis?

A crucial point to our discussion is whether migration in the water column by Paranais litoralis occurs at all in the field. We did find worms in the water-column samples from May 1995, and these worms were significantly longer than worms found in the sediment, similar to the situation we found for 'migrators' in our laboratory experiments. On average, swimming worms found in the field had 35.2 segments, which is not significantly different from laboratory migrators, but significantly longer than for non-swimming worms found in the field and in laboratory cultures. We have also found worms in colonization plates placed on the sediment surface (Nilsson et al. unpubl. obs.).

\section{Behavioral and morphological characteristics of migrators}

Paranais litoralis is a deposit-feeder, and moves through the sediment as it feeds. In this way, it has a natural dispersing mechanism without any swimming stage. Is the difference between the movement while burrowing and the swimming behavior we describe here just quantitative? Dingle (1996) argues that migration is specialized behavior especially evolved for moving to new places. Several characteristics of the swimming worms indicate that they are specialized for migration. Both in the field and in the laboratory, migrator worms were longer and thinner than non-
Table 2. Paranais litoralis. Time spent in water column. ANCOVA showing effect of worm type (migrator or nonmigrator, fixed factor), length (covariate) and time actively spent swimming (covariate)

\begin{tabular}{|lrrrr|}
\hline Source of variation & df & \multicolumn{1}{c}{ MS } & \multicolumn{1}{c|}{$F$} & \multicolumn{1}{c|}{$\mathrm{p}$} \\
\hline Worm type & 1 & 0.976 & 2.90 & 0.093 \\
Swimming activity & 1 & 11.737 & 34.89 & $<0.001$ \\
Length & 1 & 1.446 & 4.30 & 0.041 \\
Residual & 76 & 0.336 & & \\
\hline
\end{tabular}

migratory worms. This may simply be a consequence of swimming itself: worms have to stretch to swim. However, the difference in the number of segments (migrators have about $40 \%$ more segments) indicates that the shape difference is real. Being long and thin has consequences from a hydrodynamic point of view, as a long thin rod has more hydrodynamic drag than a short and wide rod of equal volume, and therefore is more easily transported passively by water currents. The effect of body shape on actively swimming animals of the size of $P$. litoralis is difficult to analyze, because the Reynolds number (based on length and swimming speeds measured) for swimming $P$. litoralis is about $\operatorname{Re}=1$, a magnitude at which neither viscous nor inertial forces predominate. In our still-water experiments, both swimming activity and large size were important for staying suspended: when worms were swimming, long worms stayed suspended significantly longer than small worms. This means that there are at least 2 components to becoming a swimming migrator: a morphological difference and a behavioral difference. Furthermore, swimming morphs forego asexual reproduction and grow to a larger size instead. From this point of view, there is a qualitative difference between dispersal via burrowing and swimming. Our interpretation is that dispersal via burrowing is a necessary consequence of the feeding mechanism of $P$. litoralis, while swimming is a change of behavior with the purpose of moving the individual to a new place as a response to an external cue (resource exhaustion).

\section{Is migration a favorable trait?}

We cannot say if the occurrence of Paranais litoralis in the water column in the field is due to active swimming or passive resuspension. Armonies (1994) found $P$. litoralis in the water column over an intertidal mud flat in Germany, and he suggested that this was due to active emergence from the sediment. Certainly many worms were actively swimming in our experiments, especially when the population was beginning to crash owing to exhaustion of resources. P. litoralis is confined 
to the oxic top surface layer of sediment, so if current speeds are high it may be difficult for worms to avoid being swept away by sediment resuspension. However, the mud flat in Flax Pond, where we collected our material, is probably a low-energy environment: tracks in the sediment sometimes remain visible for several tidal cycles, indicating that sediment resuspension is not extensive, at least not during spring and summer. Voluntary and involuntary resuspension could provide some of the advantages that active swimmers have: resuspension would deposit worms in areas where sediment is also deposited, which presumably are areas where food resources are more likely to be abundant. On the other hand, passive resuspension would give less control over time of emergence and time of swimming, and thus increase the risk of being washed away from favorable habitats. Migration of meiofauna is often an active process in sheltered habitats such as mud flats in salt marshes (Palmer 1988), but migration of sediment-burrowing invertebrates may also be a mix of local and regional processes (Armonies 1994, Palmer et al. 1996). The most favorable strategy may be to combine active behavior with passive drift: the animal may actively emerge into the water column, passively float with the current, and then actively swim down to probe the sediment, as has been described for both stream and marine invertebrates (André et al. 1993, Commito et al. 1995, Palmer et al. 1996).

We expected migrators to be in better physiological condition than non-migrators, which would give the former a reproductive advantage when they were transferred to high-quality sediment. We found no significant differences, however, between migrators and non-migrators in age at first reproduction or number of offspring produced, although the statistical power is low as our sample size was small (a second set of experiments failed as the cultures became anoxic). In nature, however, only migrators are likely to find good sediment at all. Paranais litoralis is able to completely exploit the resources of a patch, and therefore they must leave the exploited patch, making the migrator trait essential for survival. Worms that stay behind will have little chance of reproduction unless new resources are added to the patch. Those new resources must come soon; if the worms starve for long, they cannot be revived to reproduction by new resources (Levinton \& Stewart 1988, Nilsson et al. 1997). The fact that worms could choose 'good' sediment over 'exhausted' sediment is therefore crucial to the success of migrators.

Population densities of Paranais litoralis in Flax Pond peak during early summer (Giere \& Pfannkuche 1982, Cheng et al. 1993, Nilsson et al. 1997), and we did find worms in water samples in May 1995. Armonies (1994) similarly found $P$. litoralis in the water column in early summer. Late summer and early autumn may be a critical period for populations of $P$. litoralis, because resources are apparently exhausted or at least the sediment is unsuitable for growth (e.g., Cheng et al 1993). The chances of finding patches of good sediment nearby is probably low during this period, and it is therefore important to be able to migrate long distances. Because swimming is likely to lead to increased predation, the probability of actually finding suitable new sediment must be sufficiently high to offset this increased predation risk in addition to the mortality from settling in an unsuitable patch. In late summer, the number of potential competitors, e.g., the polychaetes Nereis succinea and various spionids (Levin et al. 1987, Zajac 1991) is high, and the number of potential predators (juvenile fishes, crustaceans) is also at its yearly maximum. Migrators are probably less favored during spring, when resources are still plentiful and growth can be supported in local patches.

\section{Consequences for population biology}

Several characteristics of the biology of Paranais litoralis contribute to making migration favorable: (1) complete exhaustion of resources followed by population crashes; (2) ability to choose new habitats during migration; (3) asexual reproduction decreasing the risk of extinction during an initial colonization phase. We therefore argue that $P$. litoralis is an example of an organism for which migration is not only favorable, but also essential for the survival of populations. Are the rates of movement, as measured in our diffusion experiments, fast enough to make any difference to the colonizing ability of $P$. litoralis? The asymptotic velocity of invasion of an exponentially growing population spreading as a travelling wave into a new habitat is $\sqrt{4 r D}$, where $r$ is the growth rate at low population density, and $D$ is the diffusion coefficient (Holmes et al. 1994). Applying this to $P$. litoralis would give an eventual population invasion rate of $5 \mathrm{~cm} \mathrm{~d}^{-1}$ in all directions for burrowers, and $15 \mathrm{~cm} \mathrm{~d}^{-1}$ for swimmers. From early March to late June (100 d), the offspring from a single worm spreading as burrowers could colonize an area of about $80 \mathrm{~m}^{2}$, while swimming migrators could theoretically occupy an area of $600 \mathrm{~m}^{2}$. This is calculated from rates of movement measured in still water in our experiments. The rate of movement (and hence spread) of swimming worms is probably much higher in moving water in the field. Thus, the difference in colonizing ability between worms burrowing and worms swimming is considerable. Assuming an average water depth in Flax Pond at the time of sampling in May 1995 of $0.5 \mathrm{~m}$, approximately $1 \%$ of the total population of worms (calculated from the number of 
worms per area unit in sediment samples plus water samples) would be found in the water, and this proportion would easily suffice to spread the population over the entire mud flat. Previous studies (Giere \& Pfannkuche 1982 and references therein) have shown that $P$. litoralis is very hard to find during winter, but extremely abundant during spring. There are several possible explanations for this: the worms burrow deep in the sediment during winter, they enter some sort of resting stage (e.g., overwintering cocoons), or simply their densities are so low during winter that they are unlikely to be found. The population growth rates we have found previously (Nilsson et al. 1997), and the rates of movement found in the present study indicate that while a winter resting stage is still possible, it is not necessary for explaining the seasonal pattern of $P$. litoralis. A few randomly scattered individuals at the start of the spring will suffice to colonize the entire mud flat in one season.

Acknowledgements. This study was supported by a National Science Foundation grant to J.S.L. Contribution number 1077 from the Program in Ecology and Evolution, State University of New York at Stony Brook.

\section{LITERATURE CITED}

André C, Jonsson PR, Lindegarth M (1993) Predation on settling bivalve larvae by benthic suspension feeders: the role of hydrodynamics and larval behavior. Mar Ecol Prog Ser 97:183-192

Armonies W (1994) Drifting meio- and macrobenthic invertebrates on tidal flats in Königshafen: a review. Helgol Meeresunters 48:299-320

Berg HC (1993) Random walks in biology. Princeton University Press, Princeton

Cheng IJ, Chang PC (1999) The relationship between surface macrofauna and sediment nutrients in a mudflat in Chuwei mangrove forest, Taiwan. Bull Mar Sci 65: 603-616

Cheng IJ, Levinton JS, McCartney M, Martinez D, Weissburg MJ (1993) A bioassay approach to seasonal variation in the nutritional value of sediment. Mar Ecol Prog Ser 94: $275-285$

Commito JA, Currier CA, Kane LR, Reinsel KA, Ulm IM (1995) Dispersal dynamics of the bivalve Gemma gemma in a patchy environment. Ecol Monogr 65:1-20

Dias PC (1996) Sources and sinks in population ecology. Trends Ecol Evol 11:326-330

Dingle H (1996) Migration. The biology of life on the move. Oxford University Press, New York

Giere O (1993) Meiobenthology. The microscopic fauna in

Editorial responsibility: Otto Kinne (Editor),

Oldendorf/Luhe, Germany aquatic sediments. Springer-Verlag, Berlin

Giere O, Pfannkuche O (1982) Biology and ecology of marine Oligochaeta, a review. Oceanogr Mar Biol Annu Rev 20: 173-308

Harrison S, Hastings A (1996) Genetic and evolutionary consequences of metapopulation structure. Trends Ecol Evol 11:180-183

Holmes EE, Lewis MA, Banks JE, Veit RR (1994) Partial differential equations in ecology: spatial interactions and population dynamics. Ecology 75:17-29

Hughes RN (1989) A functional biology of clonal animals. Chapman \& Hall, London

Lasserre P (1975) Clitellata. In: Giese AC, Pearse JS (eds) Reproduction of marine invertebrates, Vol III. Annelids and echiurans. Academic Press, New York, p 215-275

Levin LA, Caswell H, DePatra KD, Creed EL (1987) Demographic consequences of larval development mode: planktotrophy vs. lecithotrophy in Streblospio benedicti. Ecology 68:1877-1886

Levinton JS, Stewart S (1988) Effects of sediment organics, detrital input, and temperature on demography, production, and body size of a deposit feeder. Mar Ecol Prog Ser 49:259-266

Martinez DE (1993) On senescence in asexual metazoans. PhD thesis, Department of Ecology and Evolution, State University of New York, Stony Brook, New York

Nilsson P, Kurdziel JP, Levinton JS (1997) Heterogeneous population growth, parental effects and genotype-environment interactions of a marine oligochaete. Mar Biol 130:181-191

Nunney L, Campbell KA (1993) Assessing minimum viable population size: demography meets population genetics. Trends Ecol Evol 8:234-239

Okubo A (1980) Diffusion and ecological problems: mathematical models. Springer-Verlag, Berlin

Palmer MA (1988) Dispersal of marine meiofauna: a review and conceptual model explaining passive transport and active emergence with implications for recruitment. Mar Ecol Prog Ser 48:81-91

Palmer MA, Allan JD, Butman CA (1996) Dispersal as a regional process affecting the local dynamics of marine and stream invertebrates. Trends Ecol Evol 11:322-326

Sokal RR, Rohlf FJ (1981) Biometry. The principles and practice of statistics in biological research, 2 nd edn. Freeman \& Co, New York

Sugg DW, Chesser RK, Dobson FS, Hoogland JL (1996) Population genetics meets behavioral ecology. Trends Ecol Evol 11:338-342

Von Ende CN (1993) Repeated-measures analysis: growth and other time-dependent measures. In: Scheiner SM, Gurevitch J (eds) Design and analysis of ecological experiments. Chapman \& Hall, New York, p 113-137

Winer BJ, Brown DR, Michels KM (1991) Statistical principles in experimental design. McGraw-Hill, Inc, New York

Zajac RN (1991) Population ecology of Polydora ligni (Polychaeta: Spionidae). II. Seasonal demography variation and its potential impact on life history evolution. Mar Ecol Prog Ser 77:207-220

Submitted: February 11, 2000; Accepted: June 27, 2000 Proofs received from author(s): October 26, 2000 